\title{
The Coverage Problem in Three-Dimensional Wireless Sensor Networks
}

\author{
Chi-Fu Huang, Yu-Chee Tseng, and Li-Chu Lo \\ Department of Computer Science and Information Engineering \\ National Chiao-Tung University, Hsin-Chu, 30050, Taiwan \\ E-mail: \{cfhuang, yctseng, lolc\}@csie.nctu.edu.tw
}

\begin{abstract}
One of the fundamental issues in sensor networks is the coverage problem, which reflects how well a sensor network is monitored or tracked by sensors. In this paper, we formulate this problem as a decision problem, whose goal is to determine whether every point in the service area of the sensor network is covered by at least $\alpha$ sensors, where $\alpha$ is a given parameter and the sensing regions of sensors are modeled by balls (not necessarily of the same radius). This problem in a $2 \mathrm{D}$ space is solved in [1] with an efficient polynomial-time algorithm (in terms of the number of sensors). In this paper, we show that tackling this problem in a 3D space is still feasible within polynomial time. The proposed solution can be easily translated into an efficient polynomial-time distributed protocol.
\end{abstract}

\section{INTRODUCTION}

The rapid progress of wireless communication and embedded micro-sensing MEMS technologies has made wireless sensor networks possible. Such environments may have many inexpensive wireless nodes, each capable of collecting, storing, and processing environmental information, and communicating with neighboring nodes. In the past, sensors are connected by wire lines. Today, this environment is combined with the novel ad hoc networking technology to facilitate intersensor communication [2], [3]. The flexibility of installing and configuring a sensor network is thus greatly improved. Recently, a lot of research activities have been dedicated to sensor networks, including design issues related to the physical and media access layers [4], [5], [6] and routing and transport protocols [7], [8], [9]. Localization and positioning applications of wireless sensor networks are discussed in [10], [11], [12].

Since sensors may be spread in an arbitrary manner, two fundamental issues in a wireless sensor network are the coverage problem and the deployment problem. Given a sensor network, the coverage problem is to determine how well the sensing field is monitored or tracked by sensors, while the deployment problem is to address how to place sensors into a sensing field to meet certain coverage requirements. In the literature, these two problems have been formulated in various ways. In computational geometry, a circle covering [13] is an arrangement of circles on a plane that can fully cover the plane. The goal is to minimize the radius of circles, given a fixed number of circles. This issue is discussed in [14], [15] for square planes. Another related computational geometry issue is the Art Gallery Problem [16] which is to determine the number of observers such that every point in the art gallery is monitored by at least one observer. Reference [17] proposes polynomial time algorithms to find the maximal breach path and the maximal support path that are least and best monitored in the sensor network. How to find the minimal and maximal exposure path that takes the duration that an object is monitored by sensors is addressed in [18], [19]. On the other hand, some works are targeted at particular applications, but the central idea is still related to the coverage issue. For example, to reduce sensors' onduty time, those sensors that share the common sensing region and task may be turned off to conserve energy and thus to extend the network lifetime [20], [21], [22]. Placement of static sensors is discussed in [23], [24], while how to achieve better deployment of mobile sensors is addressed by [25], [26].

In this paper, we consider a general geometric problem related to the coverage and deployment issues: Given a set of sensors in a 3D sensing field, we want to determine if this field is sufficiently $\alpha$-covered, where $\alpha$ is a given integer, in the sense that every point in the field is covered by at least $\alpha$ sensors. The sensing range of each sensor is modeled by a 3D ball. While most applications may require $\alpha=1$, applications requiring $\alpha>1$ may occur in situations where a stronger environmental-monitoring or fault-tolerating capability is desired, such as military applications. Some applications may require multiple sensors to detect an event. For example, the triangulation-based positioning protocols [11], [12] require at least three sensors (i.e., $\alpha \geq 3$ ) at any moment to monitor a moving object.

The 2-dimensional coverage problem has been solved efficiently in [1] with a polynomial time algorithm, in terms of the number of sensors. At the first glance, the 3-dimensional coverage problem seems very difficult since even determining the subspaces divided by the spheres of sensors' sensing ranges is very complicated. However, in this paper, we show that tackling this problem is still feasible within polynomial time. Reference [21] also considers the same coverage problem in a $2 \mathrm{D}$ space combined with the communication connectivity issue. However, it incurs higher computational complexity to determine the network's coverage level compared to [1]. The arrangement issue [27], [28], which is widely studied in combinatorial and computational geometry, also considers how a finite collection of geometric objects decomposes a space into connected elements. However, to construct arrangements, only centralized algorithms are proposed in the literature, 
whilst what we need for a wireless sensor network is a distributed solution. The solution proposed in this paper can be easily translated to a distributed protocol where each sensor only needs to collect local information to make its decision.

We propose a novel solution by reducing the geometric problem from a $3 \mathrm{D}$ space to a $2 \mathrm{D}$ space, and further to a 1D space, thus leading to a very efficient solution. In essence, our solution tries to look at how the sphere of each sensor's sensing range is covered. As long as the spheres of all sensors are sufficiently covered, the whole sensing field is sufficiently covered. To determined whether each sensor's sphere is sufficiently covered, we in turn look at how each spherical cap and how each circle of the intersection of two spheres is covered. By stretching each circle on a 1-dimensional line, the level of coverage can be easily determined.

This paper is organized as follows. Section II gives some preliminaries. Our solution is presented in Section III. Section IV draws our conclusions.

\section{PReliminaries And PROBlem Statement}

We are given a set of sensors, $S=\left\{s_{1}, s_{2}, \ldots, s_{n}\right\}$, in a three-dimensional cuboid sensing field $A$. Each sensor $s_{i}, i=$ $1 \ldots n$, is located at coordinate $\left(x_{i}, y_{i}, z_{i}\right)$ inside $A$ and has a sensing range of $r_{i}$. So each sensor $s_{i}$ 's sensing area is a ball centered at $\left(x_{i}, y_{i}, z_{i}\right)$ with radius $r_{i}$, denoted as $B_{i}=$ $\left(x_{i}, y_{i}, z_{i}, r_{i}\right)$. The sphere of $B_{i}$ is the surface of $B_{i}$, denoted as $S_{i}$

Consider two sensors $s_{i}$ and $s_{j}$ which have non-empty intersecting sensing regions. The spherical cap $\operatorname{Cap}(i, j)$ is the intersection of sphere $S_{i}$ and ball $B_{j}$. The circle $\operatorname{Cir}(i, j)$ is the intersection of spheres $S_{i}$ and $S_{j}$. The center of spherical cap $\operatorname{Cap}(i, j)$, denoted by $\operatorname{Cen}(i, j)$, is the intersection of line $\overleftrightarrow{s_{i} s_{j}}$ and spherical cap $\operatorname{Cap}(i, j)$. Given any two points $p$ and $p^{\prime}$ on $S_{i}$, the geodesic distance between $p$ and $p^{\prime}$, denoted by $G D\left(p, p^{\prime}\right)$, is the minimum great circle distance between $p$ and $p^{\prime}$ on $S_{i}$. The radius of $\operatorname{Cap}(i, j)$, denoted by $\operatorname{Rad}(i, j)$, is $G D(\operatorname{Cen}(i, j), p)$, where $p$ is any point on $\operatorname{Cir}(i, j)$. Examples of these terms are illustrated in Fig. 1.

Definition 1: A point in $A$ is said to be covered by $s_{i}$ if it is within $s_{i}$ 's sensing range. A point in $A$ is said to be $\alpha$-covered if it is covered by at least $\alpha$ distinct sensors.

Definition 2: Given a natural number $\alpha$, the $\underline{\alpha}$ - $\underline{\text { Ball-Coverage }}$ $(\alpha-B C)$ Problem is a decision problem whose goal is to determine whether all points in $A$ are $\alpha$-covered or not.

\section{The Proposed Solution}

In the section, we propose an algorithm to solve the $\alpha$ BC problem with time complexity $O\left(n d^{2} \log d\right)$, where $d$ is the maximum number of sensors whose sensing ranges may intersect a given sensor's sensing range. Our approach does not try to look at how each point (or subspace) in $A$ is covered by sensors because determining how $A$ is divided by $n$ spheres is much too complicated. Instead, our algorithm tries to determine whether the sphere of a sensor under consideration is sufficiently covered. Further, to determined whether each sensor's sphere is sufficiently covered, we look at how the

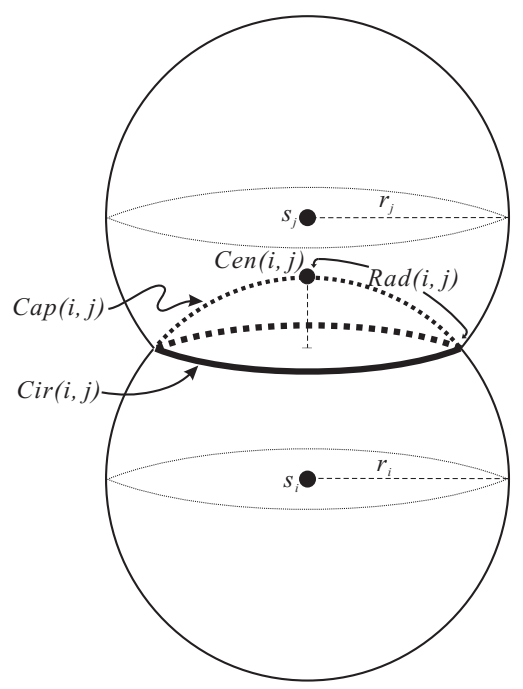

Fig. 1. Illustration of terminologies.

circle of each spherical cap of a sensor intersected by its neighboring sensors is covered. By collecting this information from all sensors, a correct answer can be obtained. Intuitively, we reduce the decision problem from a $3 \mathrm{D}$ space to one in a $2 \mathrm{D}$ space, and then to one in a $1 \mathrm{D}$ space.

\section{A. Theoretical Fundamentals}

Observe that the sensing field $A$ is divided into a number of subspaces by sensors' spheres. Each subspace's surface consists of a number of spherical segments. Because of the continuity nature, the level of coverage of a subspace can actually be derived from those of its spherical segments. Furthermore, each spherical segment must be bounded by a number of circle segments on some spherical caps. By the continuity nature again, the level of coverage of a spherical segment can actually be derived from those of its circle segments that bound the spherical segment. This is how we reduce the problem from a $3 \mathrm{D}$ space to a $2 \mathrm{D}$ space, and then to a 1D space. In the following discussion, we will use "subspace", "spherical segment", and "circle segment" to facilitate our presentation.

Definition 3: Consider any two sensors $s_{i}$ and $s_{j}$. A point on sphere $S_{i}$ is sphere-covered by $s_{j}$ if it is on or within sphere $S_{j}$. We say that $s_{i}$ is $\alpha$-sphere-covered if all points on sphere $S_{i}$ are sphere-covered by at least $\alpha$ other sensors.

Lemma 1: If a sphere $S_{i}$ is $\alpha$-sphere-covered, then each subspace that is adjacent to $S_{i}$ is at least $\alpha$-covered.

Proof: Since sphere $S_{i}$ is $\alpha$-sphere-covered, by definition each subspace that is adjacent to $S_{i}$ but outside $S_{i}$ is also $\alpha$ covered. The subspaces inside $S_{i}$ are at least $(\alpha+1)$-covered because they are further covered by $s_{i}{ }^{1}$.

Theorem 1: If each sphere is $\alpha$-sphere-covered, then the sensing field $A$ is $\alpha$-covered.

\footnotetext{
${ }^{1}$ In most cases, the subspaces inside $S_{i}$ are $(\alpha+1)$-covered. However, in the special case that there are $k$ other sensors colocating with $s_{i}$ and having the same sensing radiuses with $s_{i}$, these subspaces will be $(\alpha+k+1)$-covered.
} 


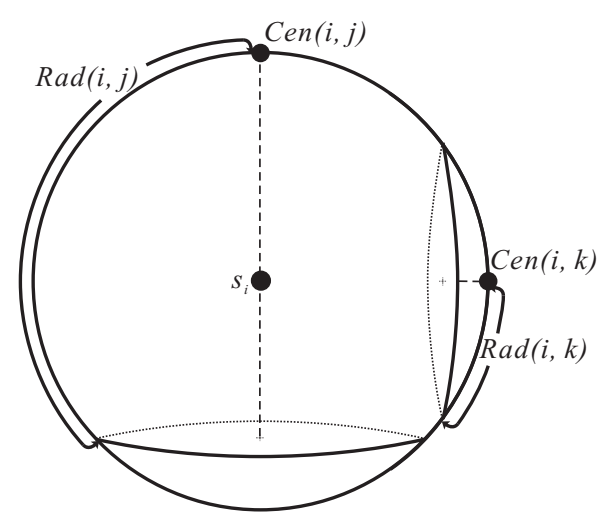

(a)

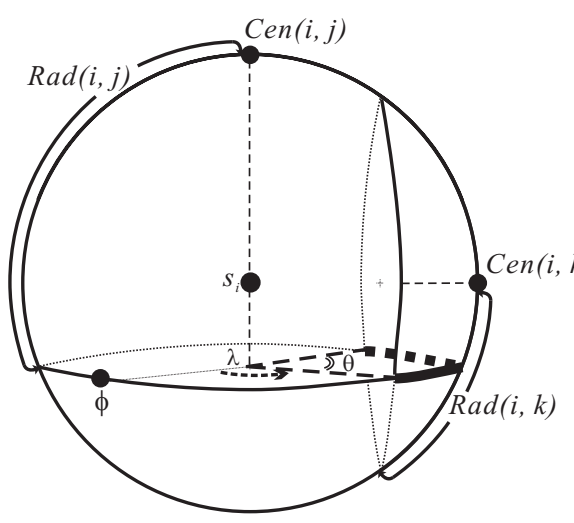

(b)

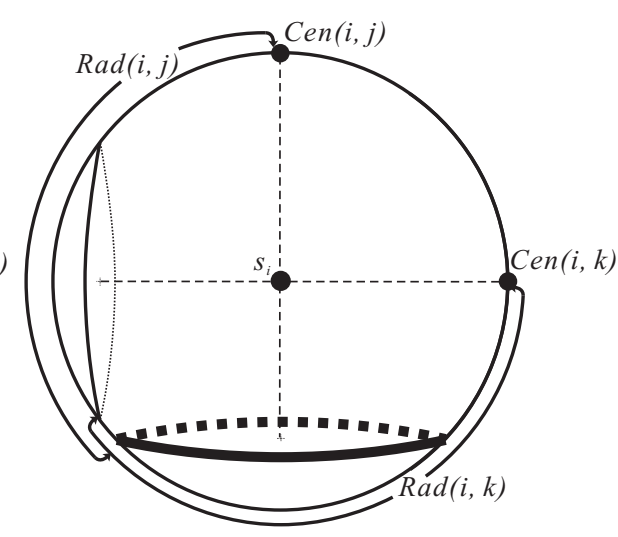

(c)

Fig. 2. The relationship between $C a p(i, j)$ and $C a p(i, k)$ : case 1 .

Proof: Observe that each subspace in $A$ must be bounded by some spherical segments. Since each sphere is $\alpha$-spherecovered, by Lemma 1 all subspaces are at least $\alpha$-covered, which proves this theorem.

Below, to facilitate our presentation, we translate sphere coverage into cap coverage. This allows us to look at a single sphere when examining coverage.

Definition 4: Consider any sensor $s_{i}$ and its neighboring sensor $s_{j}$. A point $p$ on $S_{i}$ is cap-covered by $C a p(i, j)$ if $p$ is on $\operatorname{Cap}(i, j)$. Point $p$ is $\alpha$-cap-covered if it is cap-covered by at least $\alpha$ caps on $S_{i}$.

Corollary 1: Consider any sensor $s_{i}$. If each point on $S_{i}$ is $\alpha$-cap-covered, then sphere $S_{i}$ is $\alpha$-sphere-covered.

Proof: This corollary can be easily proved by observing the equivalence between the definitions of sphere coverage and cap coverage.

Definition 5: Consider any sensor $s_{i}$ and two of its neighboring sensors $s_{j}$ and $s_{k}$. We say that a point $p$ on $\operatorname{Cir}(i, j)$ is circle-covered by $\operatorname{Cap}(i, k)$ if $p$ is cap-covered by $\operatorname{Cap}(i, k)$. We say that the spherical circle $\operatorname{Cir}(i, j)$ is $\alpha$-circle-covered if every point on $\operatorname{Cir}(i, j)$ is circle-covered by at least $\alpha$ caps on $S_{i}$ other than $C a p(i, j)$.

Lemma 2: Consider any sensor $s_{i}$ and its neighboring sensor $s_{j}$. If circle $\operatorname{Cir}(i, j)$ is $\alpha$-circle-covered, then each spherical segment on $S_{i}$ that is adjacent to $\operatorname{Cir}(i, j)$ is at least $\alpha$-cap-covered.

Proof: Since circle $\operatorname{Cir}(i, j)$ is $\alpha$-circle-covered, each spherical segment on $S_{i}$ that is adjacent to $\operatorname{Cir}(i, j)$ but outside $\operatorname{Cap}(i, j)$ is also $\alpha$-cap-covered. The spherical segments on $S_{i}$ inside $C a p(i, j)$ are at least $(\alpha+1)$-cap-covered because they are further covered by $\operatorname{Cap}(i, j)^{2}$.

Theorem 2: Consider any sensor $s_{i}$ and each of its neighboring sensors $s_{j}$. If each circle $\operatorname{Cir}(i, j)$ is $\alpha$-circle-covered, then the sphere $S_{i}$ is $\alpha$-cap-covered.

\footnotetext{
${ }^{2}$ In most cases, these spherical segments are $(\alpha+1)$-cap-covered. However, in the special case that there are $k$ other caps colocating with the current $C a p(i, j)$, these spherical segments will be $(\alpha+k+1)$-cap-covered. Note that colocating caps may appear when two spheres intersect with another sphere on the same circle.
}

Proof: Observe that each spherical segment on $S_{i}$ must be bounded by some circle segments. Since each circle is $\alpha$ cap-covered, by Lemma 2 all spherical segments on $S_{i}$ are at least $\alpha$-cap-covered, which proves this theorem.

\section{B. Determining the Intersection of Spherical Caps}

The above derivation implies that to determine how $A$ is covered, it is sufficient to determine how each circle is covered. To determine circle coverage, consider any two spherical caps $C a p(i, j)$ and $C a p(i, k)$ on sphere $S_{i}$ of a sensor $s_{i}$. There are two cases:

1: The center of $\operatorname{Cap}(i, k), \operatorname{Cen}(i, k)$, is inside $\operatorname{Cap}(i, j)$, i.e., $G D(\operatorname{Cen}(i, j), \operatorname{Cen}(i, k)) \leq \operatorname{Rad}(i, j)$.

(i) If $\operatorname{Rad}(i, k)<\operatorname{Rad}(i, j)-G D(\operatorname{Cen}(i, j), \operatorname{Cen}(i, k))$, then $\operatorname{Cap}(i, j)$ is not circle-covered by $\operatorname{Cap}(i, k)$ (refer to Fig. 2(a)).

(ii) If $\operatorname{Rad}(i, j)-G D(\operatorname{Cen}(i, j), \operatorname{Cen}(i, k)) \leq \operatorname{Rad}(i, k) \leq$ $G D(C e n(i, j), \operatorname{Cen}(i, k))+\operatorname{Rad}(i, j)$, then the arch of $\operatorname{Cir}(i, j)$ falling in the angle $[\lambda, \lambda+\theta]$ is circlecovered by $\operatorname{Cap}(i, k)$ (refer to Fig. 2(b)).

(iii) If $\operatorname{Rad}(i, k)>\operatorname{Rad}(i, j)+G D(\operatorname{Cen}(i, j), \operatorname{Cen}(i, k))$, then the whole range $[0,2 \pi]$ of $C a p(i, j)$ is circlecovered by $\operatorname{Cap}(i, k)$ (refer to Fig. 2(c)).

2: The center of $\operatorname{Cap}(i, k), \operatorname{Cen}(i, k)$, is outside $\operatorname{Cap}(i, j)$, i.e., $G D(\operatorname{Cen}(i, j), \operatorname{Cen}(i, k))>\operatorname{Rad}(i, j)$.

(i) If $\operatorname{Rad}(i, k)<G D(\operatorname{Cen}(i, j), \operatorname{Cen}(i, k))-\operatorname{Rad}(i, j)$, then $\operatorname{Cap}(i, j)$ is not circle-covered by $\operatorname{Cap}(i, k)$ (refer to Fig. 3(a)).

(ii) If $G D(\operatorname{Cen}(i, j), \operatorname{Cen}(i, k))-\operatorname{Rad}(i, j) \leq \operatorname{Rad}(i, k) \leq$ $G D(\operatorname{Cen}(i, j), \operatorname{Cen}(i, k))+\operatorname{Rad}(i, j)$, then the arch of $\operatorname{Cir}(i, j)$ falling in the angle $[\lambda, \lambda+\theta]$ is circlecovered by $C a p(i, k)$ (refer to Fig. 3(b)). Note that it is possible that there is no intersection between $\operatorname{Cir}(i, j)$ and $\operatorname{Cir}(i, k)$, but $\operatorname{Cir}(i, j)$ is fully covered by $\operatorname{Cap}(i, k)$, as illustrated in Fig. 3(c).

(iii) If $\operatorname{Rad}(i, k)>G D(\operatorname{Cen}(i, j), \operatorname{Cen}(i, k))+\operatorname{Rad}(i, j)$, then the whole range $[0,2 \pi]$ of $\operatorname{Cir}(i, j)$ is circlecovered by $\operatorname{Cap}(i, k)$ (refer to Fig. 3(d)). 


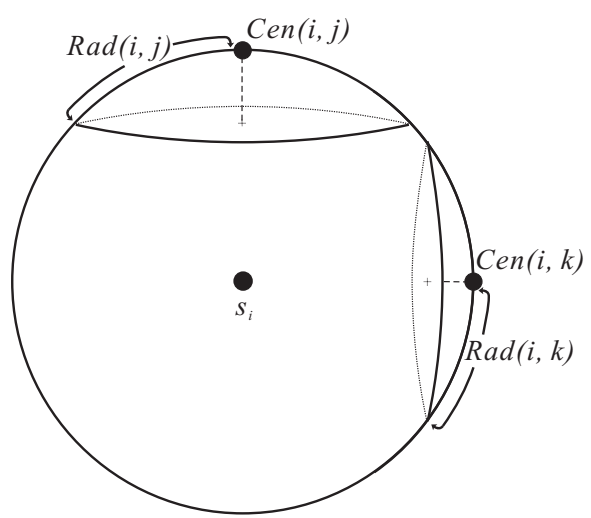

(a)

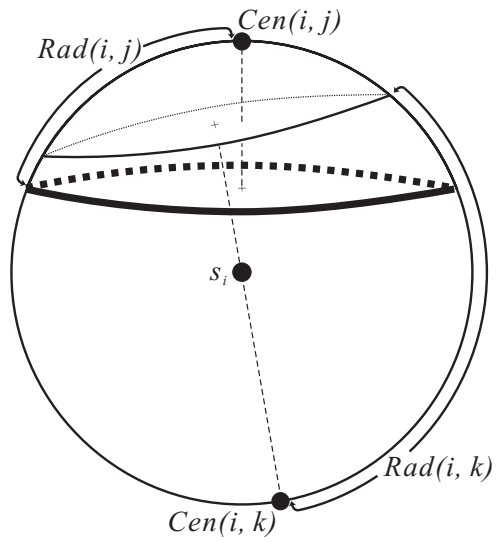

(c)

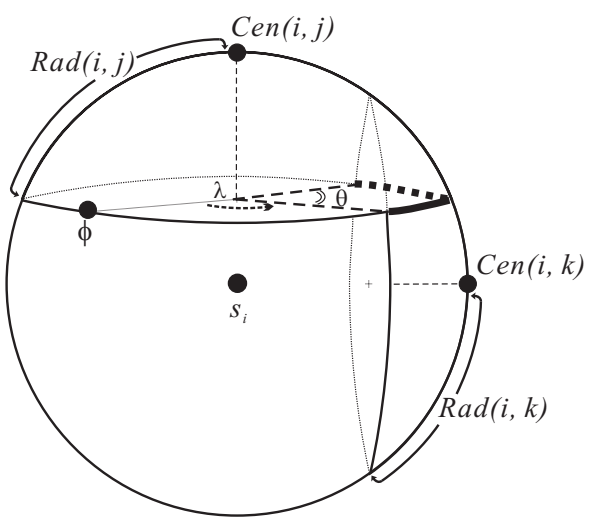

(b)

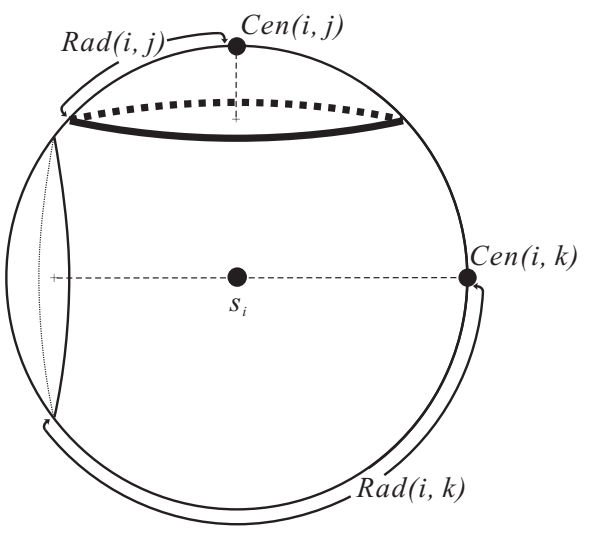

(d)

Fig. 3. The relationship between $C a p(i, j)$ and $C a p(i, k)$ : case 2 .

\section{The Complete Algorithm}

Below, we propose an $O\left(d^{2} \log d\right)$ algorithm to determine whether a sensor is $\alpha$-sphere-covered or not. The algorithm can be executed either in a centralized or in a fully distributed manner independently by each sensor. First, each sensor has to collect how its neighboring sensors intersect with itself and calculate the corresponding spherical caps. Next, it has to figure out the relationship between spherical caps, as described above. Then we can determine the level of circle coverage of each circle. After each cap's circle coverage level is determined, the sensor's sphere coverage level can be found out, which in turn gives the overall coverage of $A$. The detail algorithm to be run by each sensor $s_{i}$ is listed below.

1) For each neighboring sensor $s_{j}$ of $s_{i}$, do the following.

a) Calculate the circle $\operatorname{Cir}(i, j)$ of $\operatorname{Cap}(i, j)$.

b) For each neighbor $s_{k} \neq s_{j}$ of $s_{i}$, we determine how $\operatorname{Cap}(i, k)$ intersects with $\operatorname{Cir}(i, j)$. Specifically, we calculate the angle of $\operatorname{Cir}(i, j)$ that is circle-covered by $\operatorname{Cap}(i, k)$, denoted by $\left[\theta_{k, L}^{j}, \theta_{k, R}^{j}\right]$.

c) For all angles $\left[\theta_{k, L}^{j}, \theta_{k, R}^{j}\right]$ found in step b), place points $\theta_{k, L}^{j}$ and $\theta_{k, R}^{j}$ on a line segment $[0,2 \pi]$, and then sort all these points in an ascending order into a list $L_{j}$. d) (sketched) Traverse the line segment $[0,2 \pi]$ by sequentially visiting each point in the sorted list $L_{j}$ to determine the circle coverage of $\operatorname{Cir}(i, j)$, denoted by $c c_{j}$.

end for.

2) The sphere coverage of $s_{i}$ is the minimum circle coverage of all circles on $S_{i}$,

i.e., $\min _{\text {neighbor }} s_{j}\left\{c c_{j}\right\}$.

Let $d$ be the maximum number of sensors neighboring to a sensor $(d \leq n)$. Step 1a, 1b, 1c, and 1d have time complexities of $O(1), O(d), O(d \log d)$, and $O(d)$, respectively. So the complexity of step 1 is $O\left(d^{2} \log d\right)$, which is also the complexity of the whole algorithm for one sensor.

The step 1d, though sketched, can be easily implemented as follows. Whenever an element $\theta_{k, L}^{j}$ is traversed, the level of coverage should be increased by one. Whenever an element $\theta_{k, R}^{j}$ is traversed, the level of coverage should be decreased by one. An example is shown in Fig. 4. The point on angle 0 can be easily determined to be 3 . When visiting points $c, d$, $f, h, j, l, n$, and $p$ (resp., points $a, b, e, g, i, k, m$, and $o$ ), the level of coverage should be increased (resp., decreased) by 1 .

Below, we comment on several special cases, which we leave not addressed on purpose for simplicity in the above 


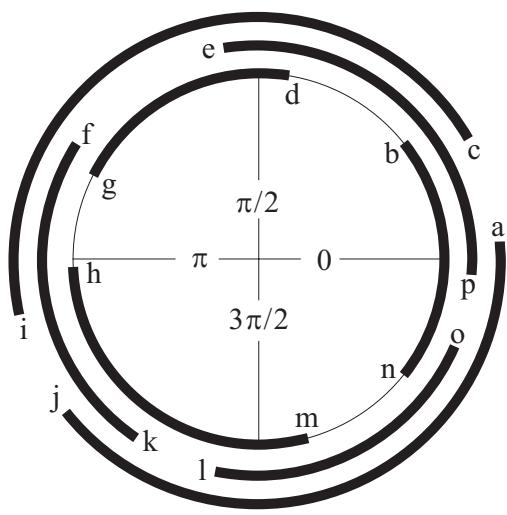

Fig. 4. An example to determine the coverage of a circle.

discussion. First, it is possible that a sensor's sensing range is fully covered by another sensor's, i.e., a sensing ball is entirely inside another sensing ball. These two spheres do not have any intersection. Alternatively, we can regard the whole sphere of the smaller one as a special spherical cap. So we can simply increase the sphere coverage level of the smaller sphere by one after executing our algorithm. Another boundary case is that some sensors' sensing ranges may exceed the sensing field $A$. If so, we can simply assign the spherical segments falling outside $A$ as $\infty$-sphere-covered.

\section{CONCLuSiOnS}

We have proposed a solution to the three-dimensional coverage problem for wireless sensor networks. We have shown that tackling this problem in a 3D space can be done at polynomial time. The result may be used in deploying sensors in 3D space and in reducing on-duty time of wireless sensors.

\section{ACKNOWLEDGMENT}

Y. C. Tseng's research is co-sponsored by the NSC Program for Promoting Academic Excellence of Universities under grant number 93-2752-E-007-001-PAE, by Computer and Communications Research Labs., ITRI, Taiwan, by Intel Inc., by the Institute for Information Industry and MOEA, R.O.C, under the Handheld Device Embedded System Software Technology Development Project and the Communications Software Technology Project, and by Chung-Shan Institute of Science and Technology under contract number BC93B12P.

\section{REFERENCES}

[1] C.-F. Huang and Y.-C. Tseng, "The coverage problem in a wireless sensor network," in ACM Int'l Workshop on Wireless Sensor Networks and Applications (WSNA), 2003, pp. 115-121.

[2] G. J. Pottie and W. J. Kaiser, "Wireless integrated network sensors," Commun. ACM, vol. 43, no. 5, pp. 51-58, May 2000.

[3] K. Sohrabi, J. Gao, V. Ailawadhi, and G. J. Pottie, "Protocols for selforganization of a wireless sensor network," IEEE Personal Commun., vol. 7, no. 5, pp. 16-27, Oct. 2000.

[4] E. Shih, S.-H. Cho, N. Ickes, R. Min, A. Sinha, A. Wang, and A. Chandrakasan, "Physical layer driven protocol and algorithm design for energy-efficient wireless sensor networks," in ACM Int'l Conf. on Mobile Computing and Networking (MobiCom), 2001, pp. 272-287.

[5] A. Woo and D. E. Culler, "A transmission control scheme for media access in sensor networks," in ACM Int'l Conf. on Mobile Computing and Networking (MobiCom), 2001, pp. 221-235.
[6] W. Ye, J. Heidemann, and D. Estrin, "An energy-efficient MAC protocol for wireless sensor networks," in IEEE INFOCOM, 2002, pp. 15671576.

[7] D. Braginsky and D. Estrin, "Rumor routing algorithm for sensor networks," in ACM Int'l Workshop on Wireless Sensor Networks and Applications (WSNA), 2002.

[8] D. Ganesan, R. Govindan, S. Shenker, and D. Estrin, "Highly resilient, energy efficient multipath routing in wireless sensor networks," $A C M$ Mobile Comput. and Commun. Review, vol. 5, no. 4, pp. 11-25, Oct. 2001.

[9] W. R. Heinzelman, A. Chandrakasan, and H. Balakrishnan, "Energyefficient communication protocols for wireless microsensor networks," in Hawaii Int'l Conf. on Systems Science (HICSS), 2000.

[10] P. Bahl and V. N. Padmanabhan, "RADAR: An in-building RF-based user location and tracking system," in IEEE INFOCOM, 2000, pp. 775784.

[11] A. Savvides, C.-C. Han, and M. B. Strivastava, "Dynamic fine-grained localization in ad-hoc networks of sensors," in ACM Int'l Conf. on Mobile Computing and Networking (MobiCom), 2001, pp. 166-179.

[12] Y.-C. Tseng, S.-P. Kuo, H.-W. Lee, and C.-F. Huang, "Location tracking in a wireless sensor network by mobile agents and its data fusion strategies," in Int'l Workshop on Information Processing in Sensor Networks (IPSN), 2003.

[13] R. Williams, The Geometrical Foundation of Natural Structure: A Source Book of Design. New York: Dover, 1979, pp. 51-52.

[14] J. B. M. Melissen and P. C. Schuur, "Improved coverings of a square with six and eight equal circles," Electronic Journal of Combinatorics, vol. 3, no. 1, 1996.

[15] K. J. Nurmela and P. R. J. Östergård, "Covering a square with up to 30 equal circles," Helsinki University of Technology, Laboratory for Theoretical Computer Science, Espoo, Finland, Research Report A62, June 2000.

[16] J. O'Rourke, "Computational geometry column 15," Int'l Journal of Computational Geometry and Applications, vol. 2, no. 2, pp. 215-217, 1992.

[17] S. Meguerdichian, F. Koushanfar, M. Potkonjak, and M. B. Srivastava, "Coverage problems in wireless ad-hoc sensor networks," in IEEE INFOCOM, 2001, pp. 1380-1387.

[18] S. Meguerdichian, F. Koushanfar, G. Qu, and M. Potkonjak, "Exposure in wireless ad-hoc sensor networks," in ACM Int'l Conf. on Mobile Computing and Networking (MobiCom), 2001, pp. 139-150.

[19] G. Veltri, Q. Huang, G. Qu, and M. Potkonjak, "Minimal and maximal exposure path algorithms for wireless embedded sensor networks," in ACM Int'l Conf. on Embedded Networked Sensor Systems (SenSys), 2003, pp. 40-50.

[20] D. Tian and N. D. Georganas, "A coverage-preserving node scheduling scheme for large wireless sensor networks," in ACM Int'l Workshop on Wireless Sensor Networks and Applications (WSNA), 2002.

[21] X. Wang, G. Xing, Y. Zhang, C. Lu, R. Pless, and C. Gill, "Integrated coverage and connectivity configuration in wireless sensor networks," in ACM Int'l Conf. on Embedded Networked Sensor Systems (SenSys), 2003, pp. 28-39.

[22] T. Yan, T. He, and J. A. Stankovic, "Differentiated surveillance for sensor networks," in ACM Int'l Conf. on Embedded Networked Sensor Systems (SenSys), 2003, pp. 51-62.

[23] K. Chakrabarty, S. S. Iyengar, H. Qi, and E. Cho, "Grid coverage for surveillance and target location in distributed sensor networks," IEEE Trans. Comput., vol. 51, no. 12, pp. 1448-1453, Dec. 2002.

[24] T. Clouqueur, V. Phipatanasuphorn, P. Ramanathan, and K. K. Saluja, "Sensor deployment strategy for detection of targets traversing a region," ACM/Kluwer Mobile Networks and Applications, vol. 8, no. 4, pp. 453461, Aug. 2003.

[25] A. Howard, M. J. Matarić, and G. S. Sukhatme, "An incremental selfdeployment algorithm for mobile sensor networks," Autonomous Robots, vol. 13, no. 2, pp. 113-126, Sep. 2002.

[26] Y. Zou and K. Chakrabarty, "Sensor deployment and target localization based on virtual forces," in IEEE INFOCOM, 2003, pp. 1293-1303.

[27] P. K. Agarwal and M. Sharir, "Arrangements and their applications," in Handbook of Computational Geometry, J.-R. Sack and J. Urrutia, Eds. North-Holland, New York: Elsevier, 2000, pp. 49-119.

[28] D. Halperin, "Arrangements," in Handbook of Discrete and Computational Geometry, J. E. Goodman and J. O'Rourke, Eds. Boca Raton, FL: CRC Press LLC, 1997, ch. 21, pp. 389-412. 